\title{
Searches for electroweak production of supersymmetric particles involving the Higgs boson and the higgsino with ATLAS
}

\section{Francisco Alonso* on behaf of the ATLAS Collaboration}

University of La Plata, IFLP, CONICET

E-mail: Francisco.alonsodcern.ch

\begin{abstract}
Fine-tuning arguments suggest the mass of the supersymmetric partner of the Higgs boson, the higgsino, is not too far from the weak scale. The search for higgsinos represents an experimental challenge due to the near mass-degeneracy resulting in soft decay products, and the low production cross section. This talk presents recent ATLAS results of analyses explicitly targeting the higgsino with a variety of experimental techniques, as well as searches for electroweak production of supersymmetric particles in final states involving the Higgs boson.
\end{abstract}

European Physical Society Conference on High Energy Physics - EPS-HEP2019 -

10-17 July, 2019

Ghent, Belgium

${ }^{*}$ Speaker. 


\section{Introduction}

Theoretical and experimental arguments suggest that the Standard Model is an effective theory valid up to a certain energy scale. The observation by the ATLAS and CMS collaborations of a particle consistent with the SM Higgs boson $(h)$ has brought renewed attention to the mechanism of electroweak symmetry breaking and the hierarchy problem: the Higgs boson mass is strongly sensitive to quantum corrections from physics at very high energy scale and demands a high level of fine-tuning. SUSY solves the hierarchy problem by introducing for each known boson or fermion a new partner (superpartner) that shares the same mass and internal quantum numbers if SUSY is unbroken. However, the superpartners have not been observed, so SUSY must be a broken symmetry and the mass scale of the sparticles is as yet undetermined. Under the R-parity conservation hypothesis, the lightest supersymmetric particle (LSP) is stable and can be a dark matter candidate. The nature of the LSP is defined by the mechanism that spontaneously breaks the supersymmetry and the parameters of the chosen theoretical framework.

The neutralinos $\left(\tilde{\chi}_{1,2,3,4}^{0}\right)$ and charginos $\left(\tilde{\chi}_{1,2}^{ \pm}\right)$represent the mass eigenstates formed from the mixture of the $\gamma, W, Z$ and Higgs bosons superpartners (the higgsinos, winos and binos). In the minimal supersymmetric extension of the SM (MSSM), the masses of the bino, wino and higgsino states are parameterised by $M_{1}, M_{2}$ and $\mu$, respectively. For large values of $\tan \beta$ these three parameters drive the phenomenology of the electroweakinos.

Naturalness and fine-tuning considerations suggest that the lightest electroweakinos (charginos and neutralinos) have masses near the electroweak scale. In such scenarios, a typical $\Delta m\left(\tilde{\chi}_{1}^{ \pm}, \tilde{\chi}_{1}^{0}\right)$ varies from a few hundred $\mathrm{MeV}$ to several tens of $\mathrm{GeV}$ depending mainly on the mass scales for the weak SUSY eigenstates. This kind of extensions to the SM including new states with nearlydegenerate masses represent an experimental challenge due to the soft decay products, and the low production cross section. Although, their direct production may be the dominant mechanism at the LHC if the superpartners of the gluon and quarks are heavier that a few TeV.

This note explores recent searches of pair production of SUSY particles via electroweak interactions explicitly targeting the higgsino, as well as searches with final states involving the Higgs boson, using data from proton-proton collisions at $\sqrt{s}=13 \mathrm{TeV}$ collected with the ATLAS detector [四].

\section{Higgsino searches in compressed scenarios}

A mass-splitting $\Delta m\left(\tilde{\chi}_{1}^{ \pm}, \tilde{\chi}_{1}^{0}\right)$ of a few $\mathrm{GeV}$ may be probed by dedicated searches requiring low-momentum leptons that arise from highly off-shell $W / Z$ bosons. In the first analysis [D] the lightest SUSY partners are assumed to be a triplet of higgsino-like states $\left(\tilde{\chi}_{1}^{0}, \tilde{\chi}_{1}^{ \pm}, \tilde{\chi}_{2}^{0}\right)$ in which the mass splitting between the states is partially determined by the magnitude of $M_{1}$ or $M_{2}$ relative to $|\mu|$. Such scenario is motivated by naturalness arguments, which suggest that $|\mu|$ should be near the weak scale while $M_{1}$ and/or $M_{2}$ can be larger. A $\tilde{\chi}_{2}^{0}$ produced in such scenario can decay to a dilepton pair via an off-shell $Z$ boson, such that the dilepton invariant mass $m_{\ell \ell}$ is kinematically restricted to be smaller than the mass splitting between the $\tilde{\chi}_{2}^{0}$ and $\tilde{\chi}_{1}^{0}$.

The analysis requires events with two same-flavour opposite-charge leptons (electrons or muons), significant missing transverse energy $\left(E_{\mathrm{T}}^{\mathrm{miss}}\right)$, and hadronic activity. Hadronic initial-state radiation 
(ISR) is also required to boost the system as a way of enhancing the sensitivity of the search. Different signal regions are defined placing additional requirements on a number of kinematic variables.

Electrons with $p_{\mathrm{T}}>4.5 \mathrm{GeV}$ and muons with $p_{\mathrm{T}}>3 \mathrm{GeV}$ are used. After all lepton selection criteria are applied, the efficiency for reconstructing and identifying signal electrons within the detector acceptance in the signal samples ranges from $20 \%$ at low $p_{\mathrm{T}}$ to $75 \%$ for $p_{\mathrm{T}}>30 \mathrm{GeV}$. The corresponding efficiency for signal muons ranges from approximately $50 \%$ at $p_{\mathrm{T}}=3 \mathrm{GeV}$ to $90 \%$ for $p_{\mathrm{T}}>30 \mathrm{GeV}$. Additionally, in order to increase the efficiency for signals with the lowest mass splittings, where the lepton $p_{\mathrm{T}}$ can be very low, signal regions based on a signal lepton and an isolated low- $p_{\mathrm{T}}$ track (with $p_{\mathrm{T}}>500 \mathrm{MeV}$ ) are used. For these regions the track is selected to be matched to a reconstructed electron or muon candidate with no identification requirements.

No significant excess was observed in any of the signal regions with respect to the expected SM background as can be seen in the left plot of Figure $\square$. There are some small deviations in some of signal regions weakening the observed limit as can be observed in the right plot of Figure $\mathbb{W}$ as a function of the $\tilde{\chi}_{2}^{0}$ mass and the mass splitting. For a mass splitting of $\sim 10 \mathrm{GeV}$ the exclusion goes up to $162 \mathrm{GeV}$ in the $\tilde{\chi}_{2}^{0}$ mass.
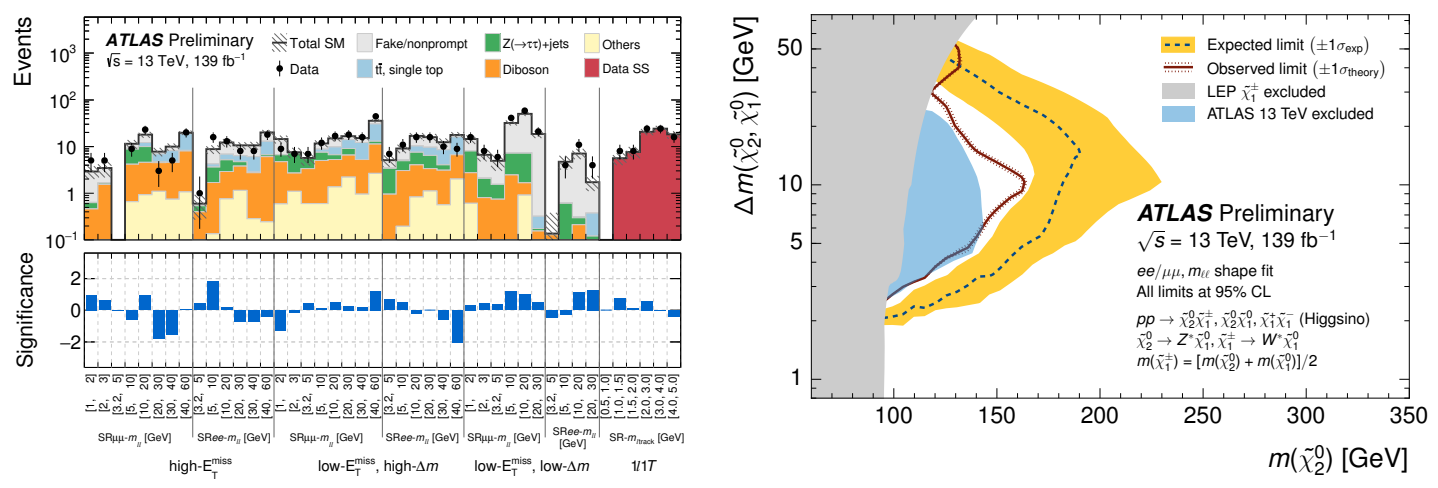

Figure 1: Left: Comparison of observed and expected event yields in the signal regions after the background-only fits. The signal regions are binned in $m_{\ell \ell}$. Uncertainties in the background estimates include both the statistical and systematic uncertainties. The bottom panel shows the significance of the difference between the expected and observed yields [च]. Right: Exclusion limits at 95\% CL for higgsino pair production $\tilde{\chi}_{1}^{+} \tilde{\chi}_{1}^{-}, \tilde{\chi}_{1}^{ \pm} \tilde{\chi}_{1}^{0}, \tilde{\chi}_{1}^{ \pm} \tilde{\chi}_{2}^{0}$, and $\tilde{\chi}_{1}^{0} \tilde{\chi}_{2}^{0}$ with off-shell SM-boson-mediated decays to the lightest neutralino, $\tilde{\chi}_{1}^{0}$, as a function of the $\tilde{\chi}_{2}^{0}$ mass and the mass splitting [[]]. The production cross-section is for pure higgsinos.

When the lightest neutralino is the LSP and it is almost pure-wino or pure-higgsino state, the mass-splitting between the LSP and the lightest chargino, $\Delta m\left(\tilde{\chi}_{1}^{ \pm}, \tilde{\chi}_{1}^{0}\right)$, is predicted to be of order $100 \mathrm{MeV}$. Probing a mass-splitting of a few hundred $\mathrm{MeV}$ is difficult for soft-lepton searches. Furthermore, the chargino becomes rather long-lived in this regime.

The lifetime of the chargino in the pure-wino (pure-higgsino) LSP scenarios is expected to be approximately $0.2(0.05) \mathrm{ns}$, such that the chargino may reach the detector before decaying. In such scenarios the chargino decays with approximately $95 \%$ branching ratio into the LSP and a soft pion, where the pion has a typical transverse momentum of $300 \mathrm{MeV}$. Since the LSP escapes detection and the pion has too low momentum to be observed, such events can be identified experimentally from the distinctive "disappearing" chargino tracks. 
The disappearing track search, which was originally designed for the long-lived wino LSP scenario with a few hundred $\mathrm{MeV}$ mass-splitting [B]], is also sensitive to the higgsino. So, this analysis [[]] is a re-interpretation of the pure-wino analysis, and is complementary to the softlepton searches. The exclusion limits can be observed together with the two lepton limits in Figure [1.

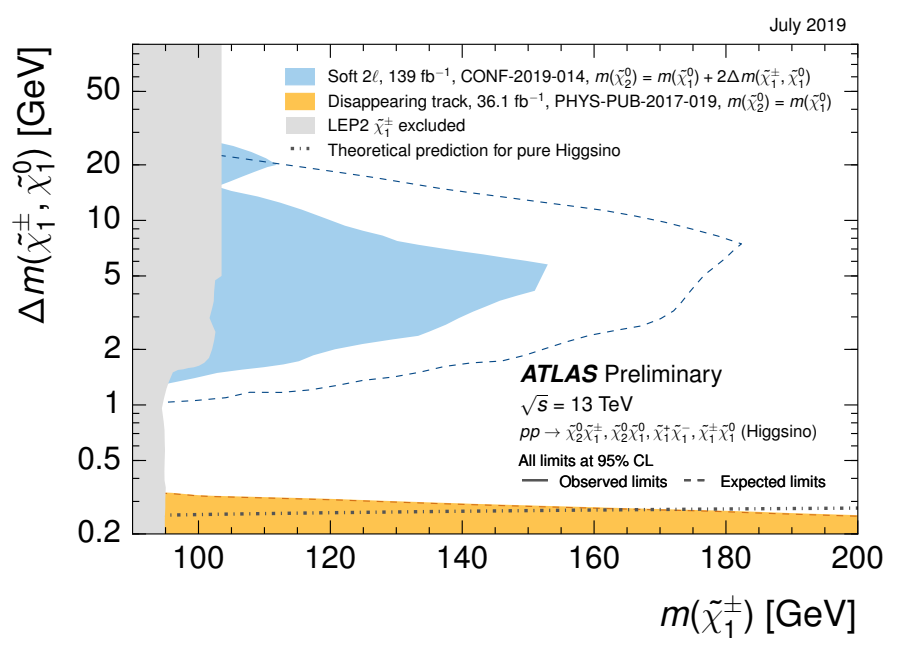

Figure 2: Summary plot including the limit for higgsino production from the two soft leptons analysis together with the limit from disappearing track analysis as a function of the $\tilde{\chi}_{1}^{ \pm}$mass and the mass splitting between $\tilde{\chi}_{1}^{ \pm}$and $\tilde{\chi}_{1}^{0}[\square]$.

\section{Searches involving the Higgs boson}

In SUSY models where the heaviest (pseudo scalar, charged) MSSM Higgs boson and the superpartners of the leptons have masses larger than those of the $\tilde{\chi}_{1}^{ \pm}$and $\tilde{\chi}_{2}^{0}$, the $\tilde{\chi}_{1}^{ \pm}$might decay into the $\tilde{\chi}_{1}^{0}$ and a $W$ boson $\left(\tilde{\chi}_{1}^{ \pm} \rightarrow W \tilde{\chi}_{1}^{0}\right)$, while $\tilde{\chi}_{2}^{0}$ could decay into $\tilde{\chi}_{1}^{0}$ and the lightest MSSM $\operatorname{Higgs}$ boson ( $h, \mathrm{SM}$-like) or $Z$ boson $\left(\tilde{\chi}_{2}^{0} \rightarrow h / Z \tilde{\chi}_{1}^{0}\right)$. The decay via the Higgs boson is dominant for many choices of the parameters as long as the mass-splitting between the two lightest neutralinos is larger than the Higgs boson mass and the higgsinos are heavier than the winos.

In ATLAS there is a comprehensive program of $W h$ searches including the following four different signatures taking advantage of using the different $W$ and Higgs decays: a) fully hadronic signature where the Higgs decays to $b b$; b) single lepton $+b$-jets signature if the $W$ decays leptonically; c) diphoton+lepton signature using the diphoton decay of the Higgs boson; d) multilepton searches including the Higgs decays to lepton through $W / Z$ or taus.

These four analyses were done with $36.1 \mathrm{fb}^{-1}$ data collected during 2015 and 2016 [四]. No significant excess was found in any of the SRs of the different channels, although there was a small excess in the diphoton SRs not allowing to set an exclusion limit. For the other channels, exclusion limits were established in neutralino and charginos masses where the high mass sensitivity is driven by both the zero lepton and one lepton channels, while the multi-lepton signature provides sensitivity in the low mas region (Figure B). 
The Wh diphoton search was updated using the full Run 2 dataset of $139 \mathrm{fb}^{-1}$ [[]], including some improvements. For the new analysis the full $W$ decays were used to improve the sensitivity. Besides, the analysis includes twelve different categories all binned in the $E_{\mathrm{T}}^{\mathrm{miss}}$ significance.

In addition to the new analysis, a follow-up of the excess observed in the previous analysis was done using the same selection as before but with the full dataset. However, no significant excess was found as seen for example in Figure

A good agreement is also observed between data and expected background in all the categories, so the exclusion limit was set using a simultaneous fit combining the different categories, providing a large gain from the previous expected limit (Figure [3).

The analysis using the leptonic decay of the $W$ and the Higgs boson decaying into $b \bar{b}$ was also performed using $139 \mathrm{fb}^{-1}$ with an improved and more powerful strategy. The three signal regions targeting the different mass splitting regions were re-optimised and now are binned in both transverse mass between the lepton and the missing transverse momentum and the contransverse mass of the two $b$-jets defined as $m_{\mathrm{CT}}=\sqrt{2 p_{\mathrm{T}}^{b_{1}} p_{\mathrm{T}}^{b_{2}}\left(1+\cos \Delta \phi_{b b}\right)}$. The background estimation strategy has been improved using a binned $t \bar{t}$ control region.

Figure $⿴$ (right) shows the agreement between data and the expected background estimates in all control, validation and signal regions. As no excess was observed in any of them, the exclusion limit is calculated using a simultaneous fit to all the multi-bin SRs, as they are orthogonal, with a important gain compared to the previous limit, as can be seen in Figure B]. The masses of electroweakinos is excluded up to $730 \mathrm{GeV}$ for the case of a mass-less $\tilde{\chi}_{1}^{0}$.

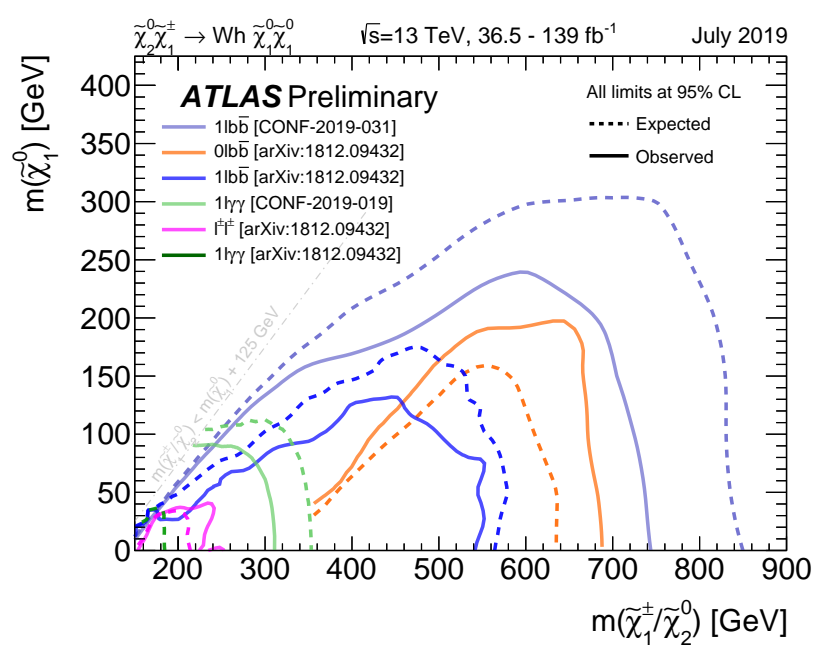

Figure 3: The $95 \% \mathrm{CL}$ exclusion limits on $\tilde{\chi}_{1}^{ \pm} \tilde{\chi}_{2}^{0}$ production with $\tilde{\chi}_{1}^{ \pm} \rightarrow \tilde{\chi}_{1}^{0} W^{ \pm}$and $\tilde{\chi}_{2}^{0} \rightarrow \tilde{\chi}_{1}^{0} h$, where $h$ is the SM-like Higgs boson, as a function of the $\tilde{\chi}_{1}^{ \pm} / \tilde{\chi}_{2}^{0}$ and $\tilde{\chi}_{1}^{0}$ masses [四]. The production cross-section is for pure wino $\tilde{\chi}_{1}^{ \pm}$and $\tilde{\chi}_{2}^{0}$.

\section{Conclusions}

There is a comprehensive electroweak search program in ATLAS involving higgsinos and the Higgs boson. Several analyses were performed probing very challenging signatures including 

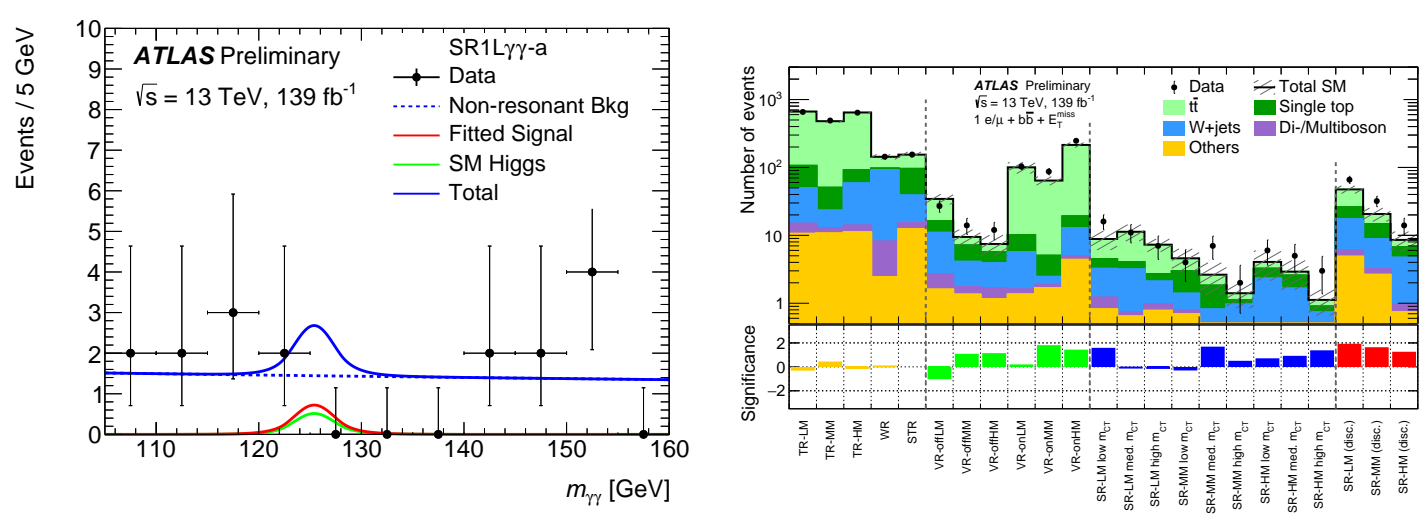

Figure 4: Left: Diphoton invariant mass spectra from data and the corresponding fitted signal and background in one of the signal regions of the $W h(\gamma \gamma)$ analysis [四]. Right: Comparison of the observed and expected event yields in control, validation and signal regions of the $W h(1 \ell b b)$ analysis. Uncertainties in the background estimates include both the statistical and systematic uncertainties [焑]. The bottom panel shows the significance of the differences between the observed and expected yields.

compressed scenarios. In all analyses a good agreement between data and the expected SM backgrounds was observed and exclusion limits in the different considered models were established with large gains with respect to previous searches due to the improved and more powerful analysis techniques used, and the full Run 2 dataset.

\section{References}

[1] The ATLAS Experiment at the CERN Large Hadron Collider, JINST 3 (2008) S08003.

[2] ATLAS Collaboration, Searches for electroweak production of supersymmetric particles with compressed mass spectra in $\sqrt{s}=13 \mathrm{TeV}$ pp collisions with the ATLAS detector, GTLAS-CONF-2019-014, CERN, Geneva, May 2019.

[3] ATLAS Collaboration, Search for long-lived charginos based on a disappearing-track signature in pp collisions at $\sqrt{s}=13 \mathrm{TeV}$ with the ATLAS detector, JHEP 06 (2018) 022 [hep-ex/1712 . 02118].

[4] ATLAS Collaboration, Search for chargino and neutralino production in final states with a Higgs boson and missing transverse momentum at $\sqrt{s}=13 \mathrm{TeV}$ with the ATLAS detector, Phys. Rev. D 100 (2019) [hep-ex/1812.09432].

[5] ATLAS Collaboration, Search for direct production of electroweakinos in final states with missing transverse energy and a Higgs boson decaying into photons in pp collisions at $\sqrt{s}=13 \mathrm{TeV}$ with the ATLAS detector, GTLAS-CONF-2019-019, CERN, Geneva, May 2019.

[6] ATLAS Collaboration, Search for direct production of electroweakinos in final states with one lepton, missing transverse momentum and a Higgs boson decaying into two b-jets in pp collisions at $\sqrt{s}=13$ TeV with the ATLAS detector, GTLAS-CONF-2019-03Д, CERN, Geneva, Jul 2019.

[7] SUSY July 2019 Summary Plot Update, GTL-PHYS-PUB-2019-022, CERN, Geneva, Jul 2019. 